\title{
Simple and Inexpensive Paper-Based Astrocyte Co-culture to Improve Survival of Low-Density Neuronal Networks
}

\author{
Mathias J. Aebersold ${ }^{\dagger}$, Greta Thompson-Steckel ${ }^{\dagger}$, Adriane Joutang, Moritz Schneider, \\ Conrad Burchert, Csaba Forró, Serge Weydert, Hana Han and János Vörös* \\ Laboratory of Biosensors and Bioelectronics, Institute for Biomedical Engineering, ETH Zürich, Zurich, Switzerland
}

\section{OPEN ACCESS}

Edited by:

Sergio Martinoia,

Università di Genova, Italy

Reviewed by:

Andreas Offenhäusser,

Forschungszentrum Jülich, Germany

Bruce C. Wheeler

University of Florida, United States

*Correspondence:

János Vörös

janos.voros@biomed.ee.ethz.ch

${ }^{\dagger}$ These authors have contributed equally to this work

Specialty section

This article was submitted to

Neural Technology,

a section of the journal

Frontiers in Neuroscience

Received: 06 November 2017

Accepted: 05 February 2018

Published: 27 February 2018

Citation:

Aebersold MJ, Thompson-Steckel G, Joutang A, Schneider M, Burchert C,

Forró C, Weydert S, Han H and

Vörös J (2018) Simple and

Inexpensive Paper-Based Astrocyte

Co-culture to Improve Survival of

Low-Density Neuronal Networks.

Front. Neurosci. 12:94

doi: 10.3389/fnins.2018.00094
Bottom-up neuroscience aims to engineer well-defined networks of neurons to investigate the functions of the brain. By reducing the complexity of the brain to achievable target questions, such in vitro bioassays better control experimental variables and can serve as a versatile tool for fundamental and pharmacological research. Astrocytes are a cell type critical to neuronal function, and the addition of astrocytes to neuron cultures can improve the quality of in vitro assays. Here, we present cellulose as an astrocyte culture substrate. Astrocytes cultured on the cellulose fiber matrix thrived and formed a dense 3D network. We devised a novel co-culture platform by suspending the easy-to-handle astrocytic paper cultures above neuronal networks of low densities typically needed for bottom-up neuroscience. There was significant improvement in neuronal viability after 5 days in vitro at densities ranging from 50,000 cells $/ \mathrm{cm}^{2}$ down to isolated cells at $1,000 \mathrm{cells} / \mathrm{cm}^{2}$. Cultures exhibited spontaneous spiking even at the very low densities, with a significantly greater spike frequency per cell compared to control mono-cultures. Applying the co-culture platform to an engineered network of neurons on a patterned substrate resulted in significantly improved viability and almost doubled the density of live cells. Lastly, the shape of the cellulose substrate can easily be customized to a wide range of culture vessels, making the platform versatile for different applications that will further enable research in bottom-up neuroscience and drug development.

Keywords: neuron, astrocyte, co-culture, paper-based, low-density culture, cell viability, neurite length, network activity

\section{INTRODUCTION}

Picking apart the immeasurable number of parallel functions occurring within the brain remains an overwhelming challenge. A complementary approach to investigating the brain in its entirety is to develop in vitro cell-based assays designed to answer a specific research question. This minimizes the many confounding variables observed in vivo. The bottom-up neuroscience approach aims to engineer well-defined cellular micro-environments in vitro to probe the fundamental mechanisms of distinct neuronal populations (Aebersold et al., 2016). By reducing the complexity, in vitro bioassays can better control experimental variables and can provide significant value for fundamental research on how the nervous system develops and functions. In addition, bottom-up neuroscience methods are a robust, versatile tool for high throughput pharmacological research and 
development of drug targets against neurodevelopmental and neurodegenerative disorders (Jones et al., 2011; Choi et al., 2013; Bicker et al., 2014; Pamies et al., 2014; Kim et al., 2015; Terrasso et al., 2015, 2017; Fukushima et al., 2016; Sandström et al., 2017).

Intensified interest in functional micro-environments has led to a reconsideration of how to design cell culture systems to increase the physiological relevance of in vitro bioassays, as it is critical that simplicity is balanced with accuracy and precision. Standard cell culture techniques can be limited by poor cell viability especially at lower cell densities, despite the access to commercially available media formulations specialized for long-term culturing of different cell types. Particular to neuro-based assays, cells must often be cultured for 2 weeks or longer to achieve connected neuronal networks that exhibit spontaneous electrophysiological activity comparable to the developing nervous system in vivo (O'Donovan, 1999). While high density neuronal cultures tend to have acceptable cell survival rates and functional activity, lower density cultures would allow for the targeting and measuring of individual cells or neurites within a defined neuronal network. Single cell and small population analysis increases the precision of experimental cause and effect compared to the complexity of network functions both in vivo and in dense in vitro cultures. There is thus a need to both increase cell survival at lower cell densities and to provide a simplified, yet physiologically relevant, micro-environment for comparable cell response in vitro.

Efforts toward meaningful cell patterning have helped to increase the physiological relevance of the experimental culture compared to organized, 3D populations in vivo (Goubko and Cao, 2009; Roy et al., 2013; Matsusaki et al., 2014; Albers et al., 2015; Tomba and Villard, 2015; Aebersold et al., 2016; Alagapan et al., 2016; Honegger et al., 2016). Techniques exist both in $2 \mathrm{D}$, with methods such as microcontact printing, and in $3 \mathrm{D}$, with the development of novel $3 \mathrm{D}$ culture substrates (Birgersdotter et al., 2005; Huh et al., 2011; Edmondson et al., 2014; Knight and Przyborski, 2015; Ravi et al., 2015; Dermutz et al., 2017). Functionalizing culture substrates with extracellular matrix proteins and other key factors is necessary not only for basic cell adhesion and viability but also for creating versatile, defined environments.

Additional efforts have been focused on recreating the composition of the extracellular environment that the experimental culture is exposed to by developing specialized synthetic media (Brewer et al., 1993, 2008) and conditioned medium (Boehler et al., 2007; Fukushima et al., 2016), or by co-culturing methods with supporting cells either directly within the culture as a feeder layer (Wang and Cynader, 1999; Yang et al., 2005; Odawara et al., 2013) or physically separated (Kaech and Banker, 2006; Fath et al., 2009; Majumdar et al., 2011; Pyka et al., 2011; Geissler and Faissner, 2012; Jones et al., 2012; Shi et al., 2013; Gottschling et al., 2016). Co-culture techniques using compartments or inserts have also successfully increased cell viability by supplementing the extracellular micro-environment without perturbing the experimental culture (Pyka et al., 2011; Dinh et al., 2013; Ehret et al., 2015; Gottschling et al., 2016).

Astrocytic conditioned medium and astrocyte co-cultures are of particular value to in vitro neuronal cultures (Banker,
1980). Astrocytes have major roles in the development, support, and maintenance of the central nervous system, with functions including the secretion of growth factors, gliotransmitters, and extracellular matrix proteins, the recycling of neurotransmitters, and the regulation of ion concentrations that affect neurotransmission (Perea et al., 2009, 2014; Clarke and Barres, 2013; Allen, 2014; Chung et al., 2015; Khakh and Sofroniew, 2015). Various studies have aimed to test neuronastrocyte interactions using in vitro co-culture systems. These have included testing for interactions during development, such as the astrocytic effect on differentiation of precursor cells and stem cells (Johnson et al., 2007; Tang et al., 2013; Ehret et al., 2015; Lischka et al., 2017; Xie et al., 2017; Schutte et al., 2018), synchronization of neuronal network activity (Kuijlaars et al., 2016), and number of formed synapses (Pyka et al., 2011; Jones et al., 2012; Shi et al., 2013), and also the effect during disease states, such as in ALS (Kunze et al., 2013), thymine deficiency (Park et al., 2001), oxidative stress (Kidambi et al., 2008), and general neurotoxicity (Anderl et al., 2009). While the relevance of co-cultures of neurons and astrocytes has become more evident as more and more functions of astrocytes are discovered, their cumulative effect on the survival of low-density networks is seldom addressed and remains unclear.

Cellulose filter paper has been demonstrated to be a mechanically stable material to use as a $3 \mathrm{D}$ culture substrate (Derda et al., 2009, 2011; Dermutz et al., 2017). The material is commercially available, inexpensive, biocompatible, bioinert, and ion and nutrient permeable (Akram et al., 2015). Various cell types, including primary neurons, readily adhere and integrate into the $3 \mathrm{D}$ porous fiber matrix, which can be functionalized with standard adhesion-promoting proteins (Derda et al., 2009, 2011; Dermutz et al., 2017). In addition, major advantages of using paper as a culture substrate are that the material is easy to handle and is compatible with standard sample preparation methods. It can also be structurally patterned via cutting, folding, rolling, and stacking, allowing for a customizable platform for versatile applications (Akram et al., 2015).

Here, we propose the use of cellulose filter paper for the construction of $3 \mathrm{D}$ primary astrocytic cultures. Because the paper substrate can easily be transferred between culture vessels, we also introduce the concept that it is an optimal co-culture platform. Astrocytes can develop separately from an experimental neuronal culture and then later be introduced by simply transferring the paper culture and suspending it over the experimental culture. With this indirect co-culture method, neuronal networks are exposed to critical secreted factors while remaining a distinguishable cell population. The supportive effect of co-culturing was assessed at a wide range of densities, from individual cells to well-connected networks, by quantifying changes in viability, neurite length and spiking activity.

\section{MATERIALS AND METHODS}

\section{Cell Culture}

All experiments were performed with primary cells from cerebral cortices of E18 embryos of time-mated pregnant Wistar rats (Harlan Laboratories, Netherlands). Animal experiments were 
approved by the Cantonal Veterinary Office Zurich. The cortices were dissociated in a $37^{\circ} \mathrm{C} / 5 \% \mathrm{CO}_{2}$ incubator for $15 \mathrm{~min}$ in $5 \mathrm{~mL}$ of a filter-sterilized solution of $0.5 \mathrm{mg} / \mathrm{mL}$ papain (P4762, SigmaAldrich, Switzerland) and 15 Kunitz units/mL deoxyribonuclease I (D5025-15KU, Sigma-Aldrich) in PBS (10010015, Gibco, Thermo Fisher Scientific, Switzerland) supplemented with $1 \mathrm{mg} / \mathrm{mL}$ bovine serum albumin (BSA, 11020021, Gibco, Thermo Fisher Scientific) and $10 \mathrm{mM} \mathrm{D-(+)-glucose} \mathrm{(G5400,} \mathrm{Sigma-}$ Aldrich). The supernatant was then removed and resuspended three times with $5 \mathrm{~mL}$ of Neurobasal medium (21103-049) supplemented with 2\% B27 (17504-044), 1\% GlutaMAX (61965026), and $1 \%$ penicillin streptomycin (15140-148; all from Thermo Fisher Scientific). The remaining solution was gently triturated to break up remaining tissue clumps. Glass $12 \mathrm{~mm}$ coverslips were activated with air plasma for 2 min (18 W PDC32G; Harrick Plasma, USA) and immediately immersed in 0.1 $\mathrm{mg} / \mathrm{mL}$ poly-D-lysine (PDL, P7280, Sigma-Aldrich) in PBS for $45 \mathrm{~min}$. Subsequently, the coverslips were washed 3 times with PBS before transfer to 24-well plates (TPP, Faust, Switzerland). For neuronal cultures, the dissociated cells were plated onto the treated coverslips at the following densities: 1,000, 2,500, $5,000,10,000,20,000$, and $50,000 \mathrm{cells} / \mathrm{cm}^{2}$. One day after cell seeding, the medium was replaced to remove any floating debris. Immediately afterwards, paper cultures were introduced to the experimental cultures designated for the co-culture condition.

For astrocyte paper cultures, instead of Neurobasal medium, cells were cultured in an astrocyte-promoting medium composed of DMEM (61965-026), 10\% fetal bovine serum (FBS, 10270106) and 1\% anti-anti (15240-062; all from Gibco, Thermo Fisher Scientific). Cells were plated at a density of 100,000 cells $/ \mathrm{cm}^{2}$ on paper substrates, prepared as described in the subsequent section.

\section{Astrocyte Feeder Cultures}

Standard Grade 2 cellulose filter papers of $8 \mu \mathrm{m}$ particle retention and $190 \mu \mathrm{m}$ thickness (Whatman, GE Healthcare, Switzerland) were cut into predefined shapes with a laser cutter (Speedy 300; Trotec Laser, Switzerland) before being air plasma-activated for $2 \mathrm{~min}$ in a polystyrene petri dish. The paper rings used for all experiments in 24-well plates had a $15.5 \mathrm{~mm}$ outer diameter and $6 \mathrm{~mm}$ inner diameter. After activation, the papers were transferred into a sterile 24-well plate under the laminar flow hood and immersed in one of the following surface functionalizing solutions: $10 \mu \mathrm{g} / \mathrm{mL}$ of laminin (L-2020, Sigma-Aldrich) in Neurobasal medium, $20 \mu \mathrm{g} / \mathrm{mL}$ of fibronectin (F1141, Sigma-Aldrich) in Neurobasal medium, or $200 \mu \mathrm{L}$ of Matrigel (Corning Matrigel Basement Membrane Matrix; 354230, Chemie Brunschwig AG, Switzerland) diluted to $2 \mathrm{mg} / \mathrm{mL}$ in ice cold serum-free medium. After $45 \mathrm{~min}$ of incubation at room temperature, the papers were rinsed with supplemented Neurobasal medium and kept in Neurobasal medium and $2 \%$ penicillin streptomycin until seeding. Controls of $12 \mathrm{~mm}$ glass coverslips were similarly plasma-activated for $2 \mathrm{~min}$ and functionalized with $10 \mu \mathrm{g} / \mathrm{mL}$ of laminin in Neurobasal medium for $45 \mathrm{~min}$.

\section{Co-culture Platform}

While paper cultures tend to float in the culture medium, they can move within the liquid and can come in contact with the substrate, in particular during manual manipulation of the well plates. To prevent direct contact, Parafilm (Parafilm, Faust) rings were attached to the side of the well plates used for experimental neuron cultures, as shown schematically in Figure 2B. Parafilm was first disinfected with $70 \%$ ethanol and then dried in the laminar flow hood. The Parafilm was then cut into bands and shaped into a ring before being placed with tweezers in a sterile 24-well plate 3 to $5 \mathrm{~mm}$ away from the bottom before cell seeding. After 1 week of culture, precursor astrocyte feeder cultures were transferred to the 1 day in vitro (DIV) experimental neuron cultures by submerging the paper in the medium using tweezers.

\section{Microcontact Printing}

To create structured networks of neurons, glass coverslips were patterned with cell-adhesive PDL on a cell-repulsive poly-Llysine grafted polyethylene glycol (PLL-g-PEG, Surface Solutions, Switzerland) background reference surface (Ricoult et al., 2014) using microcontact printing. The protocol was adapted from (Ricoult et al., 2012). Polydimethylsiloxane (PDMS; Sylgard 184, Dow Corning) stamps were replicated from an SU-8 mold and submerged in ethanol overnight after curing to extract unpolymerized PDMS oligomers. Before stamping, the stamps were disinfected by ultrasonication in $70 \%$ ethanol for $15 \mathrm{~min}$. The stamps were subsequently inked for 5 min with a solution of $10 \mu \mathrm{g} / \mathrm{mL}$ PDL and $25 \mu \mathrm{g} / \mathrm{mL}$ of Alexa Fluor 594 antibody as a fiducial marker (A-11032, Invitrogen, Thermo Fisher Scientific) in PBS. Before stamping on a plasma-activated coverslip, the stamp was rinsed for $5 \mathrm{~s}$ with PBS and then distilled water followed by drying with nitrogen. The patterned coverslip was then placed in a 24-well plate and submerged in a backfilling solution consisting of $20 \mu \mathrm{g} / \mathrm{mL}$ PLL-g-PEG in PBS for $40 \mathrm{~min}$ and finally rinsed with PBS.

\section{Immunocytochemistry}

At 14 DIV, astrocytic feeder cultures were fixed in $4 \%$ paraformaldehyde (158127, Sigma Aldrich) at room temperature for $5 \mathrm{~min}$ then blocked for $1 \mathrm{~h}$ with $3 \%$ bovine serum albumin (BSA; AlbuMAX I Lipid-Rich BSA, 11020021, Thermo Fisher Scientific) and $0.1 \%$ Triton X-100 (T-8787, SigmaAldrich) in PBS. Samples were incubated overnight at $4^{\circ} \mathrm{C}$ in 3\% BSA in PBS with the primary antibody chicken antiglial fibrillary acidic protein (GFAP; 1:500; Sigma-Aldrich) to stain for glial cells. After three times washing for $10 \mathrm{~min}$ in 3\% BSA in PBS, samples were incubated for $2 \mathrm{~h}$ in PBS with the secondary antibody goat anti-chicken IgGAlexa Fluor 568 (1:500; Thermo Fisher Scientific) and the far-red nuclei marker DRAQ5 (1:1,000, Biolegend). Samples were washed again three times for $10 \mathrm{~min}$ in PBS and then mounted onto a glass WillCo dish (WillCo Wells B. V., Netherlands). Immunocytochemistry imaging was done on a LSM 510 confocal laser scanning microscope (CLSM; Carl Zeiss, Switzerland). 


\section{Viability Analysis}

For the analysis of astrocyte viability on cellulose substrates, samples were assessed using the LIVE/DEAD Viability/Cytotoxicity Kit $(2 \mu \mathrm{M}$ Calcein $\mathrm{AM}$ and $4 \mu \mathrm{M}$ Ethidium homodimer-1, L3224, Invitrogen, Thermo Fisher Scientific in PBS) at 7 DIV. Viability of the experimental neuron cultures was measured at 5 DIV using the live/dead stain combined with a nuclear counterstain. The cultures were washed with PBS after removal of the astrocyte paper cultures for the co-culture conditions followed by $12 \mathrm{~min}$ incubation with $250 \mu \mathrm{L}$ of the live/dead stain. Next, the nuclear counterstain $(250 \mu \mathrm{L}$ of $1.62 \mu \mathrm{M}$ Hoechst 33342, H1399, Invitrogen, Thermo Fisher Scientific in PBS) was added, incubated for $10 \mathrm{~min}$, and subsequently washed with PBS.

For both astrocyte paper cultures and experimental neuron cultures, fluorescence microscopy pictures were taken of each sample at three randomized positions with an EM-CCD camera (Hamamatsu C9100-13, Hamamatsu Photonics K. K., Switzerland) on an Axio Observer Z1 inverted microscope equipped with a Colibri LED light source (Carl Zeiss, Switzerland) using $\mu$ Manager (Edelstein et al., 2014).

For image processing of astrocyte paper cultures, the number of live and dead cells was manually counted, and the three separate images per sample were summed. Due to slight autofluorescence of the cellulose paper at the excitation required to image the Hoechst stain, it was not possible to quantify the total number of cells with this method. Thus, simply the ratio of counted live cells to total number of cells was calculated.

For image processing of the experimental neuron cultures, the cells were counted using a custom Python script based on semi-automatic thresholding. The total number of cells and the number of dead cells were determined from the nuclear stain and dead stain, respectively. To calculate the viability per well, the number of live cells and dead cells of the three separate images were each summed and the ratio of live cells to total number of cells was calculated. The live stain was not used to count the number of live cells; instead the difference between total number of cells and dead cells was calculated. The density was calculated as number of live cells per imaged area.

\section{Neurite Length Analysis}

The neurite length at 5 DIV was quantified using the ImageJ (Schindelin et al., 2015) neurite tracing plugin NeuriteTracer (Pool et al., 2008). The plugin was used to calculate the overall neurite length and number of cells for each fluorescence image previously used to calculate viability. The threshold for isolation of neurites and nuclei was determined manually for one image and then applied to all images of the same condition. The results of all images per culture were then summed. To account for variation between biological replicates, the fold change in neurite length was calculated separately for each biological replicate. In detail, for each density, the values of all co-culture and control samples were divided by the mean of the control samples.

\section{Activity Analysis}

Calcium imaging was performed at 14 DIV to measure the activity. Cultures were transduced at 7 DIV with a neuronspecific AAV GCaMP6f vector using a synapsin promoter (AAV1.Syn.GCaMP6s.WPRE.SV40; Penn Vector Core, USA; Chen et al., 2013). Half of the culture medium was replaced with virus stock solution diluted 1:1,000 with fresh culture medium.

At 14 DIV, calcium activity videos were recorded with an EM$\mathrm{CCD}$ on an inverted fluorescence microscope. A video was taken at 3-5 positions per well for $2 \mathrm{~min}$ each with a frame rate of $32 \mathrm{~Hz}$. The recorded data was analyzed using a custom Python program (available at neurons.ee.ethz.ch). In brief, images are first segmented into individual cells with a consistent user-set threshold adapting for minor image variation. Next, spikes are extracted from the calcium fluorescence intensity traces using wavelet-based spike detection. For all measured cultures, the number of detected spikes in all positions was summed up to calculate the total spike frequency. The mean spike frequency per cell was calculated by dividing the total spike frequency by the number of cells detected in all positions. Analogous to the neurite length analysis, the fold change in spike frequency was calculated separately for each biological replicate.

\section{Experimental Design and Statistical Analyses}

The effect of astrocytic co-culture was assessed on culture viability, neurite length and spiking activity of primary neuron cultures plated at densities ranging from 1,000 to 50,000 cells $/ \mathrm{cm}^{2}$. To test the limit of co-culture, a lower limit of 1,000 cells $/ \mathrm{cm}^{2}$ was chosen where only negligible survival and activity was expected (Brewer, 1995). The upper limit of 50,000 cells $/ \mathrm{cm}^{2}$ was chosen as a commonly used density for in vitro experiments that reliably shows satisfactory viability and activity. All conditions were repeated with 2-3 biological replicates each consisting of 5 technical replicates. For each condition, an equal number of control experiments without astrocytic co-culture were performed. The effect of co-culture was compared to the control using the SciPy (Jones et al., 2001) implementation of the one-sided Mann-Whitney U test, which takes the non-normally distributed data into account. The reported values in Figures 1, 3, and $\mathbf{6}$ are mean \pm standard error, and in the boxplots in Figures 4, 5 the box corresponds to the first and third quartiles with the median. The ends of the whiskers are at the ends of the box, the median is indicated with a horizontal line in the box, and the maximum and minimum are at the ends of the whisker. Measurements 1.5 interquartile ranges below the first quartile or above the third quartile are classified as outliers and excluded. The reported significance levels are $p<0.05\left(^{*}\right)$, $\left.p<0.005^{(* *}\right), p<0.0005\left(^{* * *}\right)$, and $p<0.00005^{* * * *}$ ).

\section{RESULTS \\ Characterization of Astrocytic Paper-Based Culture}

Whatman filter paper was previously shown to promote extensive neuronal growth into the cellulose fiber matrix, creating a dense 
3D network especially within the Whatman Grade 2 membranes, which are characterized to have a porosity of $8 \mu \mathrm{m}$ (Dermutz et al., 2017). We adapted the culture technique for astrocytic populations by seeding primary embryonic cortical cells onto laser-cut paper membrane rings and culturing them in astrocytepromoting serum-based media. Since cellulose paper is a novel substrate for astrocytic culture, we first evaluated various surface functionalizations to optimize cell adhesion and viability. After sterilization and surface activation in a plasma cleaner, paper was submerged in a solution of laminin, fibronectin, or Matrigel to promote cell adhesion to these extracellular matrix proteins that adsorb to the paper. We compared viability and total cell count to a laminin-coated glass control at 7 days in vitro (Figures 1A-D). While astrocytes did develop on laminincoated and fibronectin-coated paper substrates, Matrigel-infused substrates held over twice as many astrocytes and had comparable viability to cells on glass controls of around 90\% (Figures 1E,F). Potential sources of the increased number of cells are improved initial adhesion, reduced cell death, and increased proliferation while in culture. All astrocyte feeder cultures further discussed were functionalized with Matrigel. The astrocytic phenotype was confirmed by immunostaining of the fixed samples targeting the astrocytic marker glial fibrillary acidic protein (GFAP) and counterstained with a nuclear marker DRAQ5, as reported in Figure 1G. Morphology and size of the GFAP-positive astrocytes were similar to those observed in other 3D substrates (East et al., 2009; Puschmann et al., 2013; Placone et al., 2015), exhibiting a lesser cell spread into three dimensions compared to the flattened, large spread of astrocytes grown on 2D glass. In addition, most of the cells in $3 \mathrm{D}$ exhibited a low level of GFAP expression characteristic of quiescent astrocytes in vivo, compared to the high level of GFAP expression typical to both $2 \mathrm{D}$ in vitro astrocytic cultures and the astrocytes in vivo that become reactive under pathological conditions (Pekny and Pekna, 2014; Hol and Pekny, 2015). Further investigations into $3 \mathrm{D}$ cell morphology were beyond the scope of the paper due to the complexity of characterizing dense 3D cultures. Paper membranes proved to be a robust, convenient platform to create astrocytic cultures that were likely more physiologically relevant than those on 2D substrates. The paper was also as easy to functionalize with adsorbed surface modifications as standard glass or tissue culture polystyrene.

\section{Effect of Co-culture on Viability}

In low-density neuronal cultures, typically below 50,000 cells $/ \mathrm{cm}^{2}$, individual cells and neurites can be easily resolved using light microscopy. Unfortunately, it is often difficult to sustain healthy cultures for more than a few days at such densities. Low viability of dissociated primary neurons has been commonly observed and reported, e.g., $65 \%$ for cortical neurons at 5,000 cells $/ \mathrm{cm}^{2}$ after 4 days in vitro (Brewer, 1995). Despite the beneficial factors provided in specialized culture media, viability is limited because neurons additionally rely on paracrine trophic factors and cell-to-cell contact, which are both minimal at lower densities (Brewer, 1995). Furthermore, mono-cultures of only neurons lack all the essential functions of astrocytes that help to create a dynamic, supportive micro-environment. Co-culturing low-density neuronal cultures with a higher density culture has been shown to be helpful in mitigating these limitations (Kaech and Banker, 2006; Fath et al., 2009). Depicted in Figure 2, we devised a novel co-culture platform to support low-density neuronal networks with astrocytes cultured on paper. Astrocyte
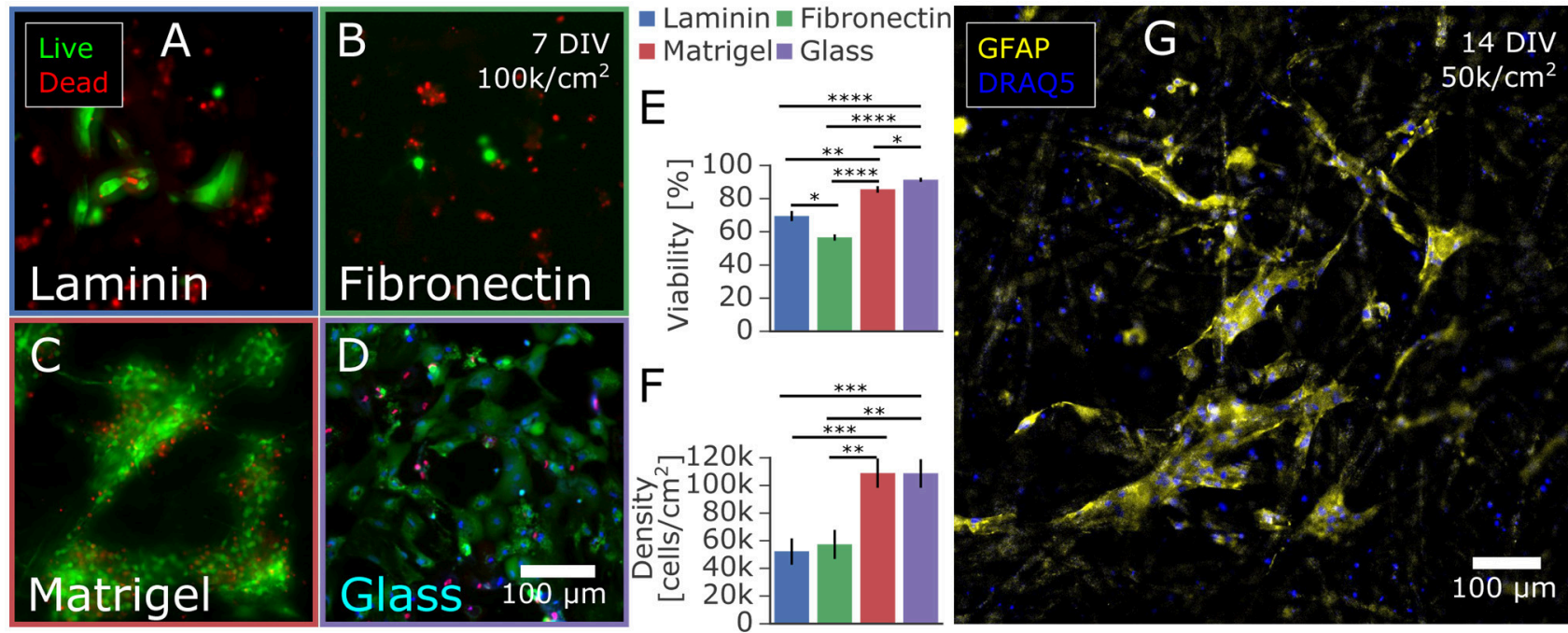

FIGURE 1 | Astrocytes isolated from primary rat cortex and cultured on paper-based substrates. (A-D) Influence of extracellular matrix protein coatings on astrocyte proliferation and viability at 7 DIV. Astrocytes were seeded at 100,000 cells/cm² on paper coated with (A) laminin, (B) fibronectin, or (C) Matrigel and on a control of (D) laminin-coated glass. After 7 days in culture with medium containing 10\% FBS, the cells were stained with a live/dead viability assay. The cells on Matrigel-coated paper had the highest (E) viability and (F) number of adhered cells likely due to proliferation and/or adhesion properties in comparison to fibronectin and laminin coated paper. (G) Immunostaining with the astrocytic marker GFAP confirmed the astrocyte phenotype of the majority of rat cortex derived cells cultured for 14 days on Matrigel coated paper. Significance levels: ${ }^{*} p<0.05 ;{ }^{* *} p<0.005 ;{ }^{* \star *} p<0.0005 ;{ }^{* * * *} p<0.00005$. 


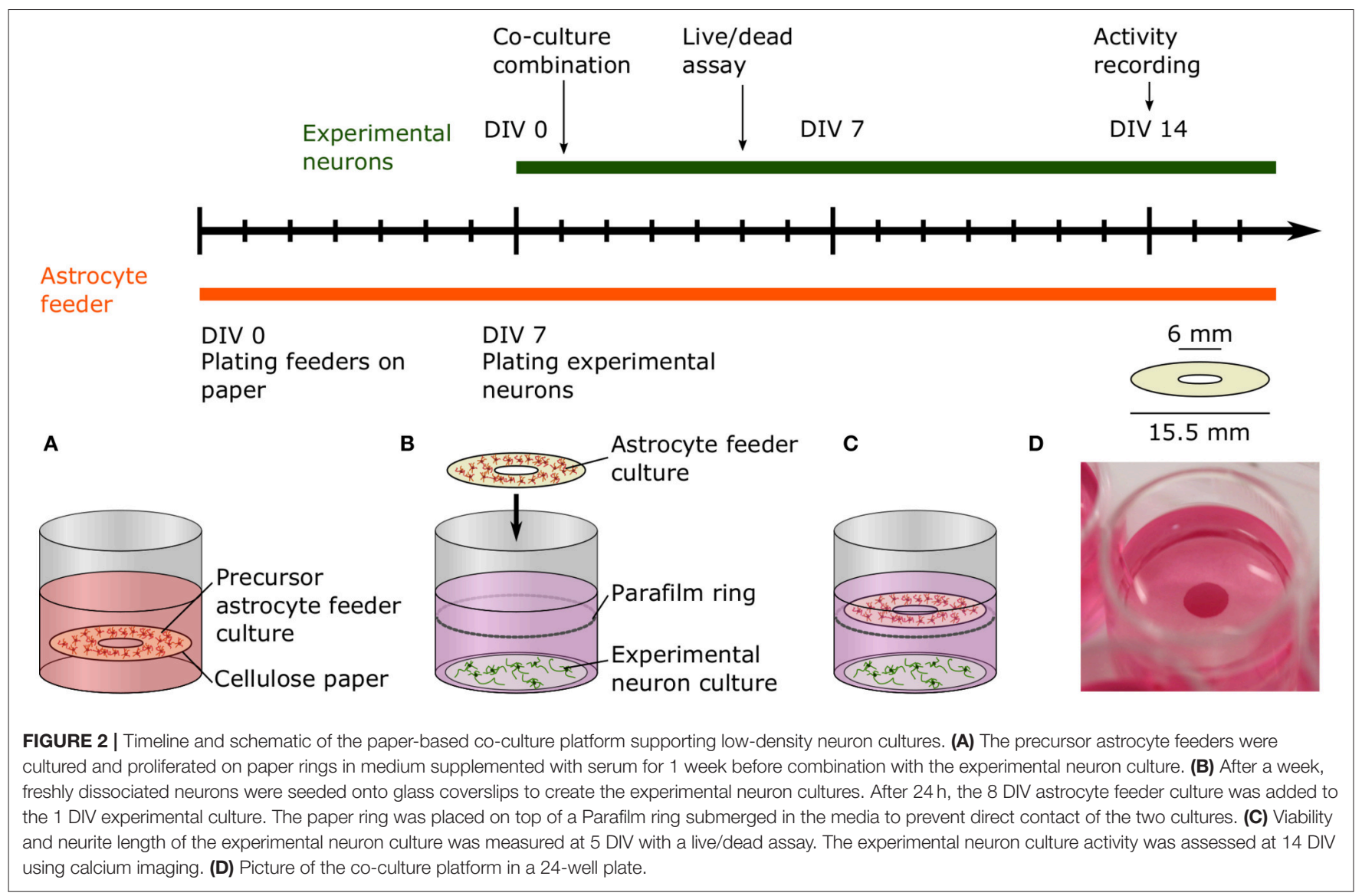

feeders are cultured separately in medium optimized for glial growth for 1 week then suspended above a 1 DIV neuronal experimental culture.

To quantify the effect of astrocytic co-culture on the survival of neuronal cultures, we cultured neurons at densities ranging from 50,000 cells $/ \mathrm{cm}^{2}$ down to cultures of isolated cells at 1,000 cells $/ \mathrm{cm}^{2}$. As a control, we repeated the same conditions but as mono-cultures without suspended astrocytic feeder cultures. In Figure 3A, there is a drastic improvement in viability across all densities compared to the control after 5 days in vitro, as measured using a live/dead assay. In the co-culture condition, viability steadily improved with increasing density until 10,000 cells $/ \mathrm{cm}^{2}$, where it then plateaued at around $90 \%$. Strikingly, viability for 1,000 cells $/ \mathrm{cm}^{2}$ was still at $70 \%$. In comparison, the control mono-culture viability mildly increased with density, albeit remaining at an overall lower viability without reaching a plateau within the range tested. Notably, at $10,000 \mathrm{cells} / \mathrm{cm}^{2}$, the control viability was only at $69 \%$ compared to $91 \%$ in the co-culture condition.

The quantification of the density of living cells at 5 DIV compared to the initial seeding density reported in Figure 3B further corroborates the supportive effect of astrocytic co-culture. In addition to the viability of the culture, this measure also took into account the commonly observed detachment of cells. Detached cells are predominantly dead, which can skew the viability obtained by a live/dead assay. Remarkably, the density of neurons supported by co-culture closely approached the theoretical limit (indicated with a dotted line), where the number of live cells at 5 DIV was equal to the number of seeded cells. Under control conditions, the resulting densities were significantly lower, with cell losses up to $50 \%$.

In Figure 3A, the value at 2,500 cells $/ \mathrm{cm}^{2}$ for the control viability was much higher than expected for this density. Unlike the viability measurement, the density of living cells is independent of dead cells. This density for the control condition at 2,500 cells $/ \mathrm{cm}^{2}$ in Figure 3B was consistent with the other conditions, suggesting that the higher viability was caused by detachment of dead cells, commonly observed during washing steps in the viability assay protocol.

These experimental results demonstrate the effectiveness of suspended astrocyte paper cultures to improve survival of lowdensity neuronal cultures. This is, to the best of our knowledge, the first quantitative assessment of the effect of an astrocytic co-culture on viability at a wide range of densities.

\section{Effect of Co-culture on Neurite Outgrowth}

While general viability is critical for a successful in vitro bioassay, a more challenging factor to tune is the functionality of the cell type to match in vivo properties. Cell morphology and outgrowth relies heavily on components within the surrounding microenvironment, which is simplified in an in vitro system. We tested whether neurons exposed to the micro-environment provided by 


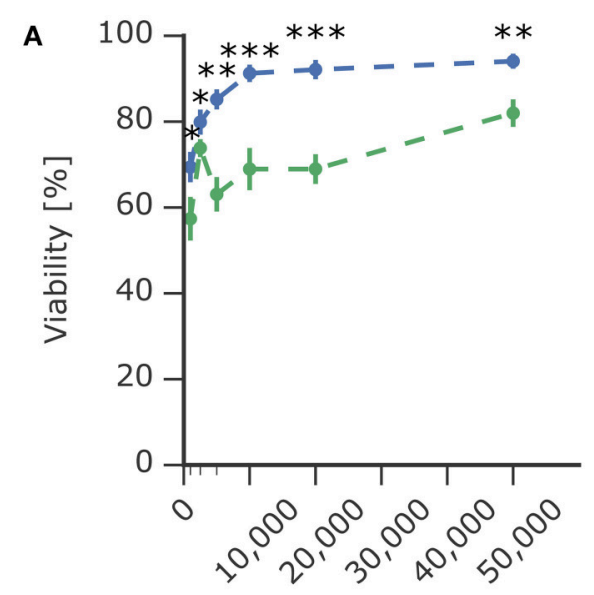

Seeding density [cells $/ \mathrm{cm}^{2}$ ]
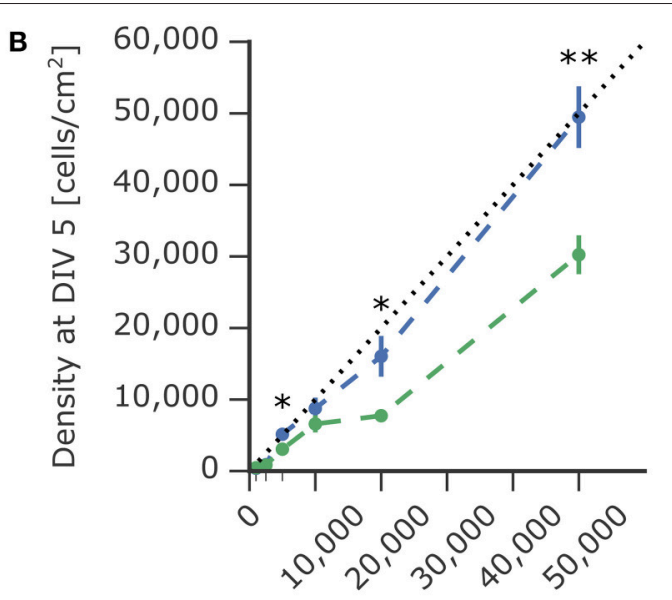

Co-culture

Control

C
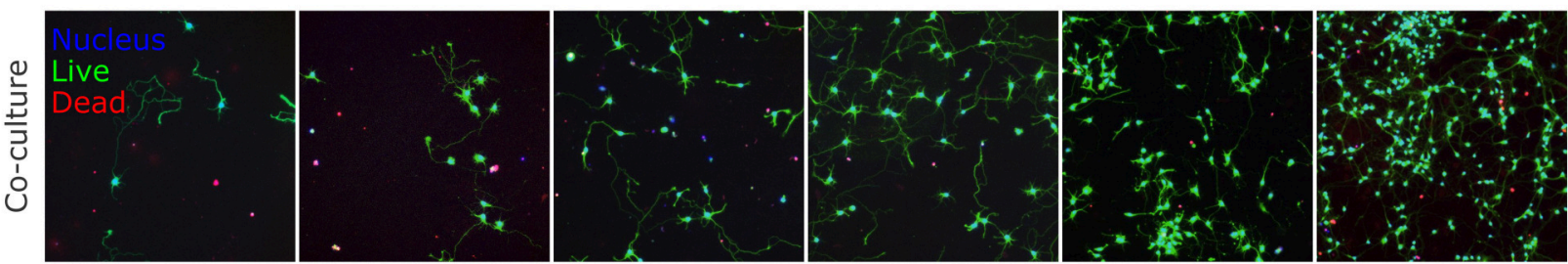

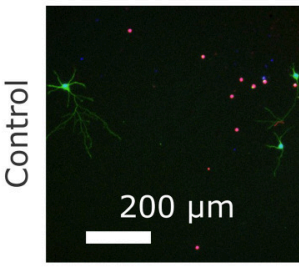

1,000

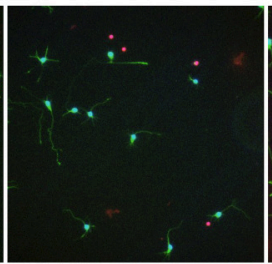

2,500

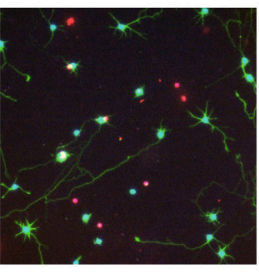

5,000

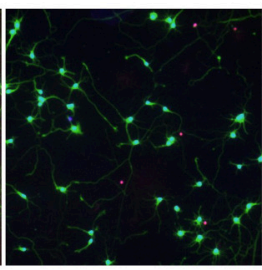

10,000

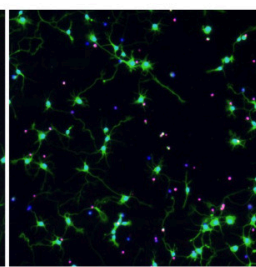

20,000

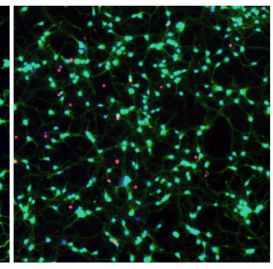

50,000

Seeding density [cells $/ \mathrm{cm}^{2}$ ]

FIGURE 3 | Effect of astrocyte co-culture on the viability of low-density neuron cultures. Primary rat cortical neurons were seeded on PDL-coated coverslips at densities ranging from 1,000 to 50,000 cells $/ \mathrm{cm}^{2}$ with paper-based astrocyte co-culture and without co-culture as a control. The viability was measured using a live/dead viability assay at 5 DIV. (A) The viability of the experimental neuron culture supported by the co-culture was significantly improved compared to the control without co-culture. (B) The density of live experimental neurons on the coverslip was calculated using the live-dead assay images and compared to the seeding density. The co-culture condition showed minimal loss of cells compared to the control condition. (C) Representative images of the experimental neuron culture with and without co-culture at different seeding densities. Plotted values are mean \pm standard error and significance was tested using Mann-Whitney $U$ test with significance levels: * if $p<0.05$; ${ }^{* *}$ if $p<0.005$; ** if $p<0.0005$.

the astrocytic co-culture showed variable developmental neurite outgrowth compared to control mono-cultures. We measured the neurite length from the fluorescent staining of cell bodies and nuclei at 5 DIV with the ImageJ Plugin NeuriteTracer. As depicted in Figure 4A, the total neurite length increased with the seeding density, as can be expected. The supportive effect of co-culturing on neurite outgrowth is visible in Figure 4B, which shows fold change increase in neurite length under coculture conditions relative to the control. While no significant increase was measured for the individual densities, an overall slight trend toward longer neurites was observed in the presence of the astrocyte paper cultures, although it is not possible to attribute the increase to a direct effect of the astrocytes on neurite length or to the overall increased viability in co-culture conditions. The extension of neurites, as characterized here, is an essential step toward forming a network of neurons, as more and longer neurites increase the chance of forming functional synaptic connections.

\section{Effect of Co-culture on Spiking Activity}

In vitro cultures of neurons spontaneously exhibit rhythmic and synchronous electrophysiological activity similar to that in developing neurons in the nervous system (Chiappalone et al., 2006, 2007). This activity is indicative of connected neuronal networks. Low-density networks, however, often do not exhibit this activity because individual cells can be isolated and not well-connected to neighboring neurons. The previously established beneficial effect of co-culturing on viability 


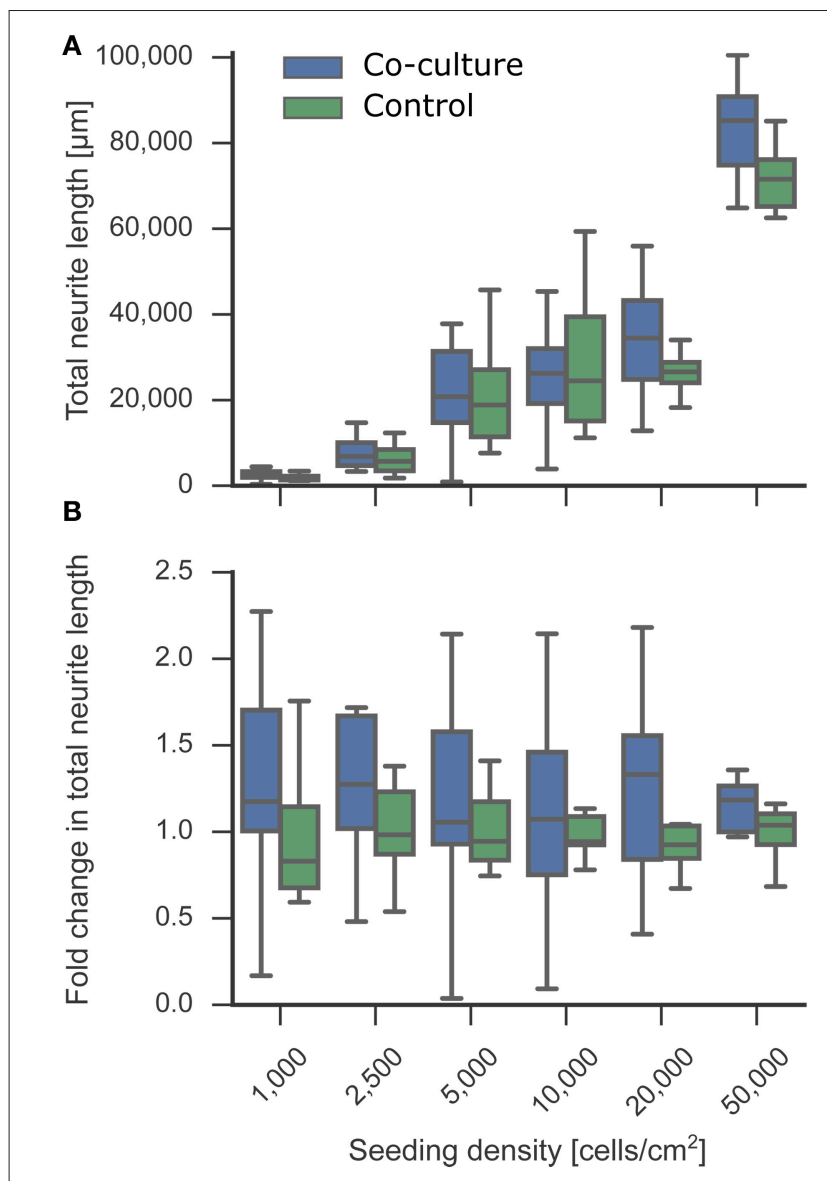

FIGURE 4 | Effect of co-culture on neurite length as an indicator for neuronal development. The neurite length of the experimental neuron cultures in co-culture and control condition was measured at 5 DIV. Tracing and quantification was done using the ImageJ Plugin NeuriteTracer. (A) Comparison of neurite lengths of the experimental neuron cultures between co-culture and control conditions. (B) The fold change of the resulting total neurite length indicated a trend toward an increased neurite length in the co-culture condition compared to the mono-culture control. In the plot, the box corresponds to the first and third quartiles with the median. The median is indicated with a horizontal line in the box, and the maximum and minimum are at the ends of the whiskers. Measurements 1.5 interquartile ranges below the first quartile or above the third quartile are classified as outliers and excluded.

and neurite outgrowth suggested a higher probability of interconnecting the sparsely distributed neurons at low densities. A connected network is required for synchronous activity, and an increased number of synapses could increase the frequency of spontaneous activity (Muramoto et al., 1993). Astrocytes also play an important role in the modulation of neuronal activity (Clarke and Barres, 2013), suggesting that co-culturing might improve electrophysiological function. Adding either astrocytes or astrocyte-conditioned medium improves the spontaneous spiking activity of neuronal cultures; however, glutamatestimulated spiking activity is increased to a considerably higher extent only when co-culturing directly with astrocytes, indicating that dynamic interactions between neurons and astrocytes are likely necessary and that conditioned medium may only have a short-lived effect (Boehler et al., 2007). We measured 2 week old neuronal cultures in co-culture and mono-culture conditions and quantified the spike frequency obtained by calcium imaging (Figure 5A). We observed spontaneous activity in all cultures down to 1,000 cells $/ \mathrm{cm}^{2}$, with the expected increase in total spike frequency at higher densities. Figure 5B demonstrates the supportive effect of astrocytic co-culturing on spiking activity based on the fold change in spike frequency compared to the control mono-culture. Co-culturing significantly increased spiking activity, most notably at all lower densities. This effect is less obvious in Figure 5A when comparing the total spike frequencies due to the high variability between biological replicates, which highlights the importance of analyzing the fold changes per biological replicate. These results may imply that the micro-environment generated by co-cultures improved neuronal signaling capabilities and increased spike frequency to more relevant levels that could be detected even in low-density cultures. This makes the suspended paper-based astrocyte cocultures platform a promising technique for in vitro assays where activity is an especially critical measurement for determining the correct functionality compared to network activity in vivo.

\section{Versatility of Co-culture Platform}

Engineering neuronal networks with well-defined topologies has sparked the community's interest for investigating the relationship between network structure and function. Patterned networks can be tailored to a specific research question with high fidelity while additionally reducing the high variability present in random, non-patterned networks. Common patterns used for network assays, such as grids and stripes, are notorious for requiring low cell densities to avoid cell clumping on the sparse pattern and thus suffer from low cell viability and lack of activity.

Microcontact printing was used to generate grid patterns of cell-adhesive poly-D-lysine on a cell-repulsive PLL-g-PEG background. We seeded cells at 10,000 cells $/ \mathrm{cm}^{2}$, which resulted in distinct networks without cell clumping but with poor viability, as evident in Figure 6A. Applying the co-culture platform presented here significantly improved the viability from $62 \%$ to $84 \%$ at 5 DIV and almost doubled the density of live cells from 3,500 cells $/ \mathrm{cm}^{2}$ to 6,700 cells $/ \mathrm{cm}^{2}$ (Figures $6 \mathrm{~B}-\mathrm{D}$ ). In addition to our co-culture substrates optimized for 24-well plates, the shape of the cellulose substrate can easily be customized to a wide range of culture vessels, including but not limited to a 96well plate, a standard commercial multielectrode array, and a T25 culture flask (Figure 6E).

\section{DISCUSSION}

The need for more physiologically relevant in vitro bioassays generates demand for a neuron-astrocyte co-culture platform that is well-characterized yet simple to use. While existing methods have been essential for advancing knowledge of neuron-astrocyte interactions, they can be cumbersome and expensive. Furthermore, they are frequently lacking general characterization, in particular in the context of low-density cultures. 

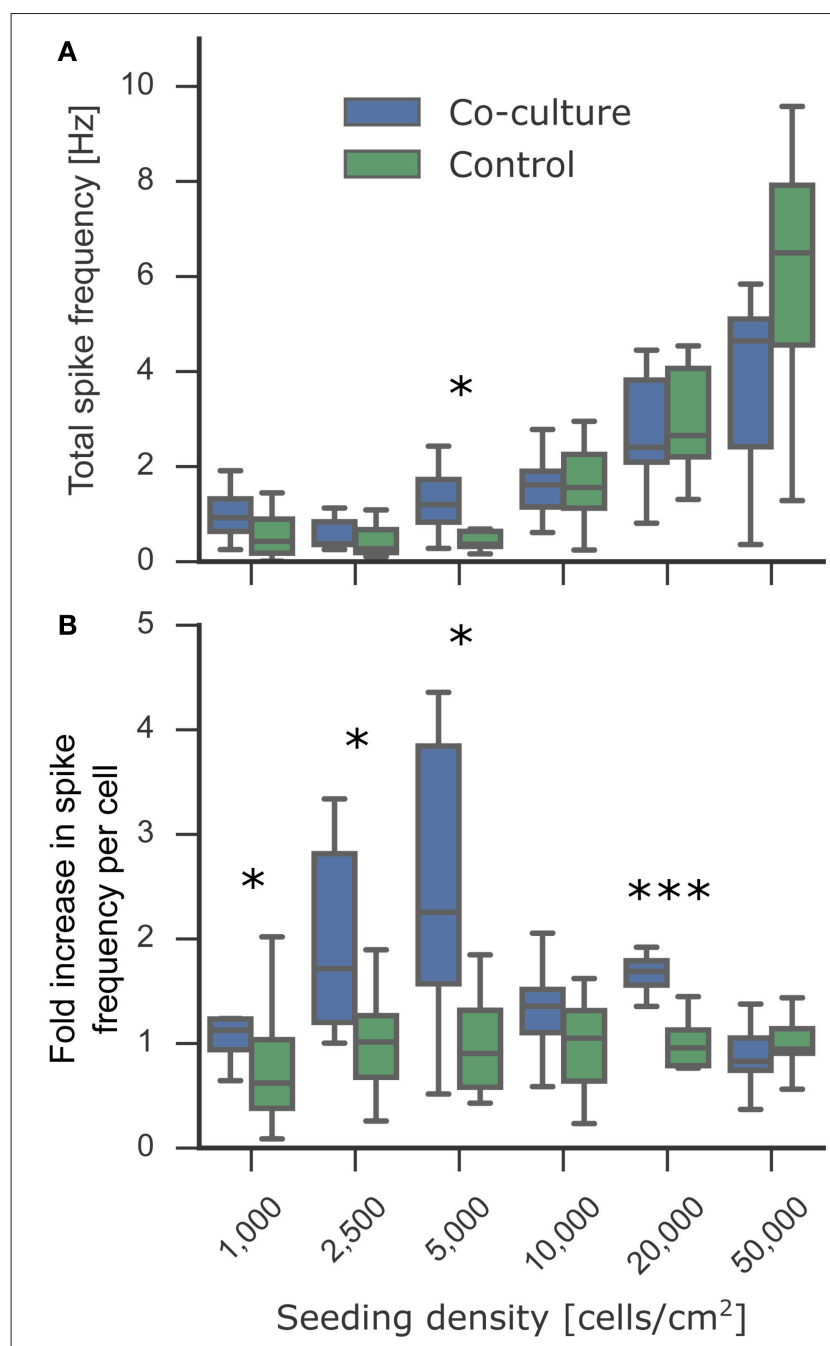

FIGURE 5 | Effect of astrocyte co-culture on the spiking activity of low-density neuronal cultures. The cultures were transduced with the gene-encoded calcium indicator GCaMP6f at 7 DIV. At 14 DIV, the activity of the experimental neuronal cultures was measured using the fluorescent calcium indicators. To obtain the spiking activity, the calcium activity videos were segmented into individual cells followed by spike detection. (A) Spontaneous activity was detected in all cultures down to $1,000 \mathrm{cells} / \mathrm{cm}^{2}$. The culture-wide spike frequency increases progressively with the seeding density. (B) The fold increase in spike frequency per neuron between the co-cultured and control experimental neuron cultures showed an increase in spike frequency at low densities. In the plot, the box corresponds to the first and third quartiles with the median. The median is indicated with a horizontal line in the box, and the maximum and minimum are at the ends of the whiskers. Measurements 1.5 interquartile ranges below the first quartile or above the third quartile are classified as outliers and excluded. Statistical significance was tested using Mann-Whitney $U$-test with significance levels: * if $p<0.05$; ${ }^{* \star \star}$ if $p<0.0005$.

\section{Characterization of Astrocytic Paper-Based Culture}

As a well-characterized cell culture substrate, glass coverslips have been used in co-culture systems by suspending them above or next to the experimental culture (Park et al., 2001; Jones et al., 2011). When suspended, glass presents a physical barrier that prevents the diffusion of essential gasses and nutrients. Culturing on the side creates a non-uniform diffusion gradient of trophic factors across the experimental culture. Cellulose paper has been established as a cell culture platform that is permeable and does not act as a complete physical barrier (Derda et al., 2009; Dermutz et al., 2017). The 3D fiber matrix also more closely resembles the physiological conditions in tissues. Furthermore, the sturdy paper can easily be handled for addition and removal at any point during the experiment. It can be shaped according to the form and dimensions of the culture vessel. These properties make paper an ideal candidate for a neuron-astrocyte co-culture platform. Bacteria and plant-based cellulose is a commonly used biomaterial (Vandamme et al., 1998), in particular as a surface coating (Missoum et al., 2013). Early studies in the 70s aimed to bridge lesions in the spinal cord with astrocyte-infused paper implants (Eng et al., 1986). In more recent work, cellulose in its paper form has been rediscovered as a convenient substrate particularly for microfluidic diagnostics and in vitro cell-based assays. Our approach presented here establishes paper as a substrate for astrocytic culture with the aim of improving viability and development of low-density neuronal cultures via co-culture.

As the first step of the optimization process, the evaluation of different surface functionalizations showed a drastic difference in performance as a substrate for astrocyte culture, as evident in Figure 1. After 7 days in culture, Matrigel-coated paper contained twice as many cells than fibronectin and laminincoated substrates in addition to significantly improved cell viability. We attribute the increased number of cells on Matrigel, a reconstituted basement membrane mixture of adhesionpromoting extracellular matrix proteins, to a combination of better adhesion after plating, survival and proliferation (Kleinman and Martin, 2005; Hughes et al., 2010; Levy et al., 2014). Notably, cellulose was compatible with standard coating methods for glass and plastic substrates. As a consequence, surface functionalization with adhesion-promoting proteins did not require significant adaptation.

While the viability and number of cells at 7 DIV of Matrigelcoated paper was comparable to the laminin-coated glass control, the cell morphology had characteristic differences between the $2 \mathrm{D}$ and $3 \mathrm{D}$ substrates, similar to as previously reported in the literature (East et al., 2009; Puschmann et al., 2013; Placone et al., 2015). Cells cultured on the cellulose fiber matrix thrived and formed a dense 3D network of small, stellate shaped cells compared to the large, flat cell structure typical of astrocytes on $2 \mathrm{D}$ glass. Immunostaining proved that the majority of cells stained specifically for GFAP, an astrocyte specific marker. In conclusion, we established Matrigel-coated paper as an astrocyte culture substrate ideally suited for co-culturing because of its permeability, ease-of-use, and 3D structure.

Designed specifically for co-culturing in 24-well plates, the ring shape of the paper allowed for convenient visual inspection through the center hole without removal of the paper, while maximizing the surface area available for astrocytes. Due to the neutral buoyancy of paper, the astrocyte cultures floated within the culture medium. However, as a preventative measure against mechanical damage to the underlying culture, in particular during transportation, a Parafilm spacer ring was added below the paper to ensure separation. Parafilm is inert 

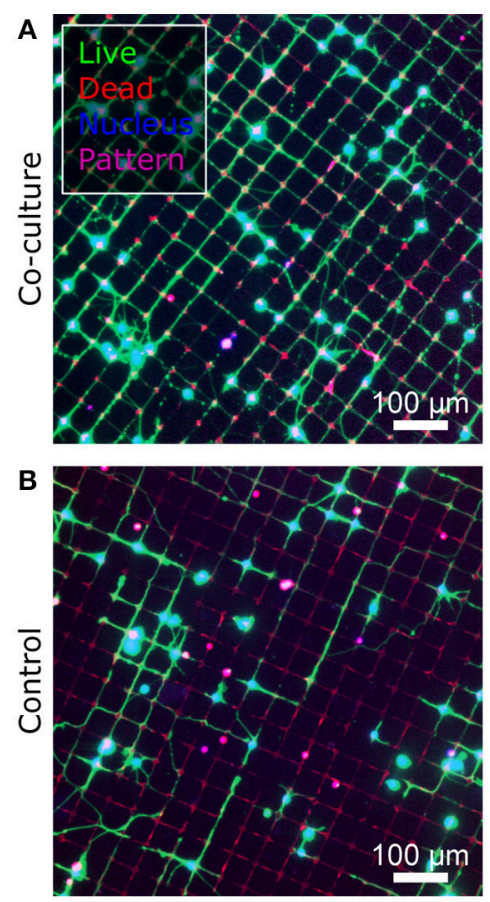
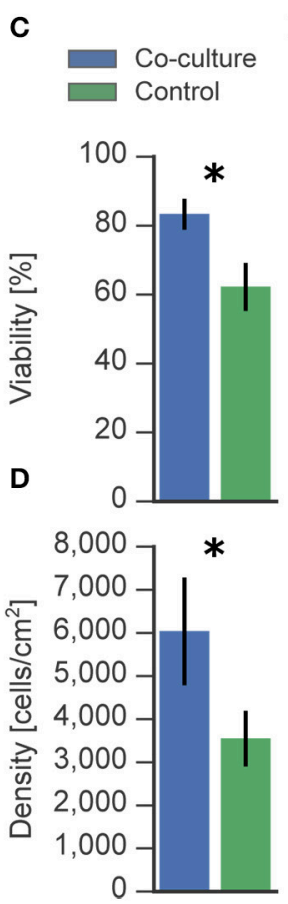
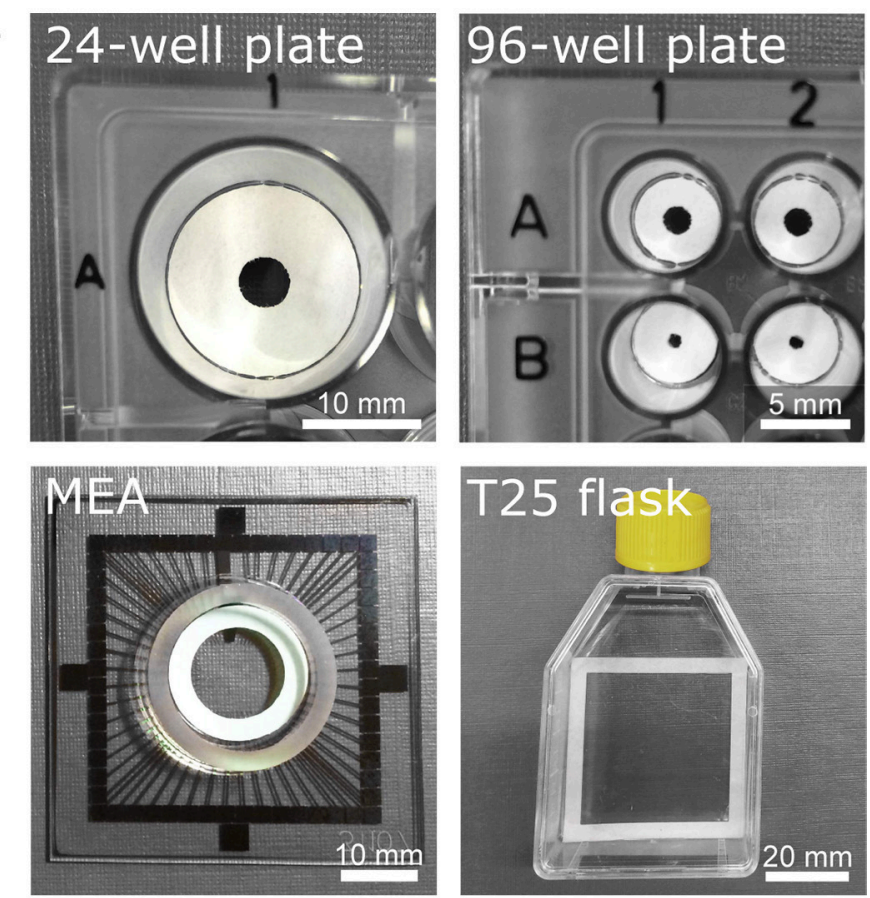

FIGURE 6 | Applications and versatility of paper-based co-culture platform. (A,B) Effect of astrocytic co-culture on low-density neurons on microcontact-printed patterns. Patterned networks often require low densities to avoid clumping of neurons on the cell-adhesive regions, resulting in low viability. (C,D) Cell viability and density was improved under co-culture condition compared to control mono-cultures. (E) Paper-based culture can be structured to fit any culture vessel, such as well plates, multielectrode arrays and flasks, for versatile applications. Significance levels: ${ }^{*} p<0.05$.

and biocompatible (Yoo and Nam, 2012) and did not interfere with the cell culture. As outlined in the experimental timeline in Figure 2, precursor astrocyte cultures were matured for 1 week prior to combination with the 1 DIV experimental neuronal cultures. At 1 week, astrocytes populated the Matrigel-paper matrix at a sufficient density to be able to provide support to the low-density neuronal cultures. The supportive effect on cell viability was first assessed after 5 DIV, after the plateauing of initial cell death common to primary neuronal cultures. The time point was also used to analyze neurite length, as there was sufficient neurite growth at 5 DIV without dense neurite overgrowth that obscures quantification. Additional experimental cultures were developed for 14 DIV with a geneencoded calcium indicator to quantify the effect of co-culturing on electrophysiological activity. At this time point, neurons have formed well-connected networks, which can exhibit spontaneous activity detectable by calcium imaging.

\section{Effect of Co-culture on Viability}

In addition to enhancing general biological relevance, astrocyte co-cultures for in vitro assays are critical for improving survival and functionality of low-density cultures, including those needed for the bottom-up neuroscientific approach of building well-defined neuronal networks. While the positive effects of astrocytes on neuron cultures are broadly accepted, their influence on viability of low-density neuronal networks has not been sufficiently examined.
Here, we show that continuous support from astrocytes achieved a remarkably high viability of above $90 \%$ for densities down to 10,000 cells $/ \mathrm{cm}^{2}$. Even for isolated cells at a density of 1,000 cells $/ \mathrm{cm}^{2}$, the effect of co-culturing was significant with a viability of $69 \%$. In contrast, mono-cultures required ten times more cells to reach this same viability. Overall, coculturing dramatically shifted the viability curve toward lower densities, achieving high viability at densities where single cells and neurites can easily be resolved and analyzed. This enables the targeting of single cells in experiments that were previously hindered by prohibitively low viability. The comparison of our results with alternative methods is difficult, as quantification across seeding densities is lacking. Nevertheless, the limited published viability measurements are comparable to our analysis (Brewer and Cotman, 1989; Lucius and Mentlein, 1995; Pyka et al., 2011).

The standard live/dead assay tends to overestimate viability because it is unable to measure the unavoidable fraction of cells that die and detach during the initial phase after cell seeding. A more complete quantification is to measure the density of live cells compared to the initial seeding density. We observed a substantial loss of cells in mono-cultures measured after 5 days. Mono-cultures seeded at 50,000 cells $/ \mathrm{cm}^{2}$ resulted in a density of only 30,000 cells $/ \mathrm{cm}^{2}$, while more than half of the cells were lost at a seeding density of 20,000 cells $/ \mathrm{cm}^{2}$. In comparison, the seeding densities and the actual densities after 5 DIV in co-cultures were closely matched, with greater than $80 \%$ 
efficiency for densities of 5,000-50,000 cells $/ \mathrm{cm}^{2}$. We attribute this higher efficiency to an improved viability induced by coculturing, which reduced the amount of detaching dead cells. Unlike conditioned medium, this platform provides a convenient way to give continuous support of potentially short-lived factors without the need of maintaining separate cultures to produce the medium and of frequently exchanging the medium.

Viability is influenced by both the surrounding microenvironment and by direct cell-cell contact (Brewer et al., 1993). The improved viability of more than $90 \%$ at 10,000 cells $/ \mathrm{cm}^{2}$ and above indicates that the physically separate co-culture can provide the appropriate micro-environment for enhanced cell survival. The change in viability below 10,000 cells $/ \mathrm{cm}^{2}$ shows the point where supporting lower density cultures with secreted factors alone is not sufficient anymore, suggesting that direct cell-cell interactions are likely the limiting factor. It remains unclear to what degree reciprocal communication has an effect on compartmentalized neurons and astrocytes in vitro, particularly since cultures lack the organized, close proximity of astrocytes at synapses that is normal within the brain. Since gliotransmission and neurotransmission are integrally coupled functions at the synapse, it is possible that only slower, long-range communication between cell types plays a significant role in these separated cultures. While direct culturing of astrocytes with neurons offers the added effect of cell-to-cell contact-mediated effects on viability, it introduces an additional layer of complexity from the multitude of inevitable interactions between the two cell populations. Thus, many in vitro assays require separation of cell types to obtain clear experimental cause and effect.

\section{Effect of Co-culture on Neurite Outgrowth}

Across all densities, neurons developed rapidly with extensive neurite outgrowth in both co-cultures and mono-cultures. The astrocyte co-cultures were added to 1 DIV neuronal cultures and were thus present during the critical period of neurite development following initial cell adhesion. Because astrocytes have been shown to positively influence neurite outgrowth (Smith et al., 1990; Filous et al., 2010), we measured the length of neurites at 5 DIV to characterize the extent of the astrocytic effect in vitro.

We observed a slight trend toward increased neurite length in co-culture conditions compared to control. The measured increase in neurite length could have resulted from an increase either in total number of neurites or in longer neurites. However, with the measurement method used, it was not possible to distinguish between the two cases. While it is also difficult to differentiate between the direct effect of astrocytes on the neurite outgrowth and the indirect effect caused by an overall higher viability, a denser network of neurites increases the chance of forming synaptic connections. This effect is particularly important in low-density cultures where individual cells are spaced far apart and are less likely to make connections and develop into active networks.

\section{Effect of Co-culture on Spiking Activity}

Studies using low-density neuronal cultures rarely investigate electrophysiological activity because of the difficulty to keep the cultures alive long-term until synapse maturation and onset of spiking activity. Neuromodulation of spiking properties has been an important experimental target for in vitro assays. Our results demonstrated the beneficial effect of co-culturing on neurite outgrowth, a crucial step toward electrophysiologically active networks. We investigated how co-culturing low-density neuronal networks with astrocytes for 2 weeks influenced their development into functionally connected and active networks. Astrocyte cultures were removed during the activity measurement to isolate the effect of co-culture on the development of the neuronal network while excluding any real-time regulatory effects. Cultures exhibited spontaneous spiking even at a very low density of 1,000 cells $/ \mathrm{cm}^{2}$, with a strong trend toward greater total spike frequencies in co-culture conditions compared to control. When comparing the average spike frequency per cell, co-culturing resulted in a significantly greater spike frequency at lower densities. Notably, with our platform, the astrocyte co-culture could alternatively remain during measurements to investigate specific neuromodulatory effects of astrocytes.

The use of a gene-encoded calcium indicator made it feasible to repeat every condition at least five times per biological replicate to minimize the variability caused by cell population heterogeneity. At such low densities, where only few cells can be measured, a sufficient sampling size is particularly necessary for adequate quantification. Nevertheless, a more in depth study of electrophysiological properties could be achieved using multielectrode arrays (MEAs), which are better for probing temporal dynamics of networks but often with the tradeoff of a lower sample size (Ito et al., 2010; Geissler and Faissner, 2012). In a previous study, the minimum density for spontaneous activity was found to be 25,000 cells $/ \mathrm{cm}^{2}$ for cortical neurons grown on a MEA in astrocytic conditioned medium (Ito et al., 2010). Even without co-culturing, we observed spiking activity below $25,000 \mathrm{cells} / \mathrm{cm}^{2}$, possibly because of the greater number of cells measured separately on account of improved spatial resolution with calcium imaging.

\section{Versatility of Co-culture Platform}

Having characterized the supportive effect of our co-culture platform, we explored the potential effect on patterned networks, a popular application deterred by low viability and activity at low densities. The supportive effect of co-culturing in random cultures was also observed on an interconnected grid of neurons. Co-cultures seeded at a density of $10,000 \mathrm{celll} / \mathrm{cm}^{2}$ reached a viability of $83 \%$ and a density of living cells of $6,000 \mathrm{cells} / \mathrm{cm}^{2}$ after 5 DIV. In comparison, the control cultures measured significantly lower with a viability of $69 \%$ and a density of 3,550 cells $/ \mathrm{cm}^{2}$, only a third of the initial seeding density and half of the co-culture. Compared to random, non-patterned cultures, the absolute viability and density values measured after 5 DIV were lower for neurons on grid patterns. The overall area for cells to adhere was reduced on the grid pattern, where the nodes are just big enough for one cell body and the thin lines can only support a few neurites. Cells that do not initially land on the pattern fail to adhere on the non-fouling background, which results in a lower cell density and thereby 
also a lower viability. In conclusion, these preliminary results with patterned networks of neurons show the potential of this co-culture platform for improving the cell culture conditions in bottom-up neuroscience.

While we have optimized the co-culture platform toward improving neuronal survival with astrocytic support, it is not limited to this application. Based on the experimental paradigm, the platform could easily be adapted for different cell types and different setups. The feeder cultures and experimental cultures can initially be kept separate, so there is complete flexibility for variations in media conditions, patterning techniques, patterns, and cell densities. Due to the ease of transferring the paper substrate, the combination and removal, if required, of the two separate cultures is simple and robust at any chosen time point. The co-culture can also be added at multiple time points, if necessary. Furthermore, the cellulose substrate is commercially available as Whatman filter paper in different forms, porosities, and thicknesses. The substrate can conveniently be adapted to fit any culture vessel by folding, rolling, or cutting. While laser cutting produces a large amount of substrate quickly, it is also possible to use a scalpel, paper puncher, and scissors. Standard surface chemistry modifications can additionally be applied to the cellulose membrane depending on what is optimal for a specific cell type. Overall, our platform is a robust, versatile approach that can easily be customized toward different experimental aims.

\section{CONCLUSION}

Here, we have introduced a reliable, robust technique to conveniently co-culture astrocytes with neuronal cultures that is simple and inexpensive. The inclusion of astrocytes enhances in vitro bio-assays due to the essential neuron-astrocyte interactions that improve neuronal survival and function. We

\section{REFERENCES}

Aebersold, M. J., Dermutz, H., Forró, C., Weydert, S., Thompson-Steckel, G., Vörös, J., et al. (2016). Brains on a chip: towards engineered neural networks. TrAC Trends Anal. Chem. 78, 60-69. doi: 10.1016/j.trac.2016. 01.025

Akram, M. S., Daly, R., da Cruz Vasconcellos, F., Yetisen, A. K., Hutchings, I., and Hall, E. A. H. (2015). "Applications of paper-based diagnostics," in Lab-on-aChip Devices and Micro-Total Analysis Systems (Cham: Springer International Publishing), 161-195.

Alagapan, S., Franca, E., Pan, L., Leondopulos, S., Wheeler, B. C., and DeMarse, T. B. (2016). Structure, function, and propagation of information across living two, four, and eight node degree topologies. Front. Bioeng. Biotechnol. 4:15. doi: 10.3389/fbioe.2016.00015

Albers, J., Toma, K., and Offenhäusser, A. (2015). Engineering connectivity by multiscale micropatterning of individual populations of neurons. Biotechnol. J. 10, 332-338. doi: 10.1002/biot.201400609

Allen, N. J. (2014). Astrocyte regulation of synaptic behavior. Annu. Rev. Cell Dev. Biol. 30, 439-463. doi: 10.1146/annurev-cellbio-100913-013053

Anderl, J. L., Redpath, S., and Ball, A. J. (2009). A Neuronal and astrocyte coculture assay for high content analysis of neurotoxicity. J. Vis. Exp. e1173. doi: $10.3791 / 1173$

Banker, G. A. (1980). Trophic interactions between astroglial cells and hippocampal neurons in culture. Science 209, 809-810. doi: $10.1126 /$ science. 7403847 quantified this supportive effect on low-density neuronal cultures at a wide range of cell seeding densities, from isolated cells to well-connected networks. In co-culture conditions, there was an increase in viability and spiking activity, in particular at low densities. Using a paper-based substrate additionally makes the platform customizable to different applications that will further enable research in bottom-up neuroscience and drug development.

\section{AUTHOR CONTRIBUTIONS}

MA, GT-S, and JV designed the research project. MA and GT-S performed and analyzed all the experiments. AJ performed preliminary experiments. $\mathrm{MA}, \mathrm{CB}$, and MS developed the calcium imaging software. $\mathrm{HH}$ produced the mold for microcontact printing. SW and CF gave critical input and discussion. MA and GT-S wrote the paper. All co-authors reviewed the paper.

\section{FUNDING}

This work was supported by the Swiss National Science Foundation and ETH Zurich.

\section{ACKNOWLEDGMENTS}

We thank Stephen Wheeler (LBB) for help with laser cutting. We would like to give special thanks to Prof. Dr. Shiva Tyagarajan, Prof. Dr. Jean-Marc Fritschy, and in particular Giovanna Bosshard from the Institute of Pharmacology and Toxicology-Morphological and Behavioral Neuroscience at the University of Zurich for generously providing us with rat cortical tissue.

Bicker, J., Alves, G., Fortuna, A., and Falcão, A. (2014). Blood-brain barrier models and their relevance for a successful development of CNS drug delivery systems: a review. Eur. J. Pharm. Biopharm. 87, 409-432. doi: 10.1016/j.ejpb.2014.03.012

Birgersdotter, A., Sandberg, R., and Ernberg, I. (2005). Gene expression perturbation in vitro-a growing case for three-dimensional (3D) culture systems. Semin. Cancer Biol. 15, 405-412. doi: 10.1016/j.semcancer.2005.06.009

Boehler, M. D., Wheeler, B. C., and Brewer, G. J. (2007). Added astroglia promote greater synapse density and higher activity in neuronal networks. Neuron Glia Biol. 3, 127-140. doi: 10.1017/S1740925X07000440

Brewer, G. J. (1995). Serum-free B27/neurobasal medium supports differentiated growth of neurons from the striatum, substantia nigra, septum, cerebral cortex, cerebellum, and dentate gyrus. J. Neurosci. Res. 42, 674-683. doi: 10.1002/jnr.490420510

Brewer, G. J., and Cotman, C. W. (1989). Survival and growth of hippocampal neurons in defined medium at low density: advantages of a sandwich culture technique or low oxygen. Brain Res. 494, 65-74. doi: 10.1016/0006-8993(89)90144-3

Brewer, G. J., Boehler, M. D., Jones, T. T., and Wheeler, B. C. (2008). NbActiv4 medium improvement to Neurobasal/B27 increases neuron synapse densities and network spike rates on multielectrode arrays. J. Neurosci. Methods 170, 181-187. doi: 10.1016/j.jneumeth.2008.01.009

Brewer, G. J., Torricelli, J. R., Evege, E. K., and Price, P. J. (1993). Optimized survival of hippocampal neurons in B27-supplemented Neurobasal, a new serum-free medium combination. J. Neurosci. Res. 35, 567-576. doi: 10.1002/jnr.490350513 
Chen, T.-W., Wardill, T. J., Sun, Y., Pulver, S. R., Renninger, S. L., Baohan, A., et al. (2013). Ultrasensitive fluorescent proteins for imaging neuronal activity. Nature 499, 295-300. doi: 10.1038/nature12354

Chiappalone, M., Bove, M., Vato, A., Tedesco, M., and Martinoia, S. (2006). Dissociated cortical networks show spontaneously correlated activity patterns during in vitro development. Brain Res. 1093, 41-53. doi: 10.1016/j.brainres.2006.03.049

Chiappalone, M., Vato, A., Berdondini, L., Koudelka-Hep, M., and Martinoia, S. (2007). Network dynamics and synchronous activity in cultured cortical neurons. Int. J. Neural Syst. 17, 87-103. doi: 10.1142/S0129065707000968

Choi, Y. J., Park, J., and Lee, S.-H. (2013). Size-controllable networked neurospheres as a 3D neuronal tissue model for Alzheimer's disease studies. Biomaterials 34, 2938-2946. doi: 10.1016/j.biomaterials.2013.01.038

Chung, W.-S., Allen, N. J., and Eroglu, C. (2015). Astrocytes control synapse formation, function, and elimination. Cold Spring Harb. Perspect. Biol. 7:a020370. doi: 10.1101/cshperspect.a020370

Clarke, L. E., and Barres, B. A. (2013). Emerging roles of astrocytes in neural circuit development. Nat. Rev. Neurosci. 14, 311-321. doi: 10.1038/nrn3484

Derda, R., Laromaine, A., Mammoto, A., Tang, S. K. Y., Mammoto, T., Ingber, D. E., et al. (2009). Paper-supported 3D cell culture for tissue-based bioassays. Proc. Natl. Acad. Sci. U.S.A. 106, 18457-18462. doi: 10.1073/pnas.0910666106

Derda, R., Tang, S. K. Y., Laromaine, A., Mosadegh, B., Hong, E., Mwangi, M., et al. (2011). Multizone paper platform for 3D cell cultures. PLOS ONE 6:e18940. doi: 10.1371/journal.pone.0018940

Dermutz, H., Thompson-Steckel, G., Forró, C., de Lange, V., Dorwling-Carter, L., Vörös, J., et al. (2017). Paper-based patterned 3D neural cultures as a tool to study network activity on multielectrode arrays. RSC Adv. 7, 39359-39371. doi: 10.1039/C7RA00971B

Dinh, N.-D., Chiang, Y.-Y., Hardelauf, H., Baumann, J., Jackson, E., Waide, S., et al. (2013). Microfluidic construction of minimalistic neuronal co-cultures. Lab. Chip 13, 1402-1412. doi: 10.1039/c3lc41224e

East, E., Golding, J. P., and Phillips, J. B. (2009). A versatile 3D culture model facilitates monitoring of astrocytes undergoing reactive gliosis. J. Tissue Eng. Regen. Med. 3, 634-646. doi: 10.1002/term.209

Edelstein, A. D., Tsuchida, M. A., Amodaj, N., Pinkard, H., Vale, R. D., and Stuurman, N. (2014). Advanced methods of microscope control using $\mu$ Manager software. J. Biol. Methods 1, 1-10. doi: 10.14440/jbm.2014.36

Edmondson, R., Broglie, J. J., Adcock, A. F., and Yang, L. (2014). Threedimensional cell culture systems and their applications in drug discovery and cell-based biosensors. Assay Drug Dev. Technol. 12, 207-218. doi: 10.1089/adt.2014.573

Ehret, F., Vogler, S., and Kempermann, G. (2015). A co-culture model of the hippocampal neurogenic niche reveals differential effects of astrocytes, endothelial cells and pericytes on proliferation and differentiation of adult murine precursor cells. Stem Cell Res. 15, 514-521. doi: 10.1016/j.scr.2015.09.010

Eng, L. F., Stöcklin, E., Lee, Y. -L., Shiurba, R. A., Coria, F., Halks-Miller, M., et al. (1986). Astrocyte culture on nitrocellulose membranes and plastic: detection of cytoskeletal proteins and mRNAs by immunocytochemistry and in situ hybridization. J. Neurosci. Res. 16, 239-250. doi: 10.1002/jnr.490160121

Fath, T., Ke, Y. D., Gunning, P., Götz, J., and Ittner, L. M. (2009). Primary support cultures of hippocampal and substantia nigra neurons. Nat. Protoc. 4, 78-85. doi: 10.1038/nprot.2008.199

Filous, A. R., Miller, J. H., Coulson-Thomas, Y. M., Horn, K. P., Alilain, W. J., and Silver, J. (2010). Immature astrocytes promote CNS axonal regeneration when combined with chondroitinase ABC. Dev. Neurobiol. 70, 826-841. doi: 10.1002/dneu.20820

Fukushima, K., Miura, Y., Sawada, K., Yamazaki, K., and Ito, M. (2016). Establishment of a human neuronal network assessment system by using a human neuron/astrocyte co-culture derived from fetal neural stem/progenitor cells. J. Biomol. Screen. 21, 54-64. doi: 10.1177/1087057115610055

Geissler, M., and Faissner, A. (2012). A new indirect co-culture set up of mouse hippocampal neurons and cortical astrocytes on microelectrode arrays. J. Neurosci. Methods 204, 262-272. doi: 10.1016/j.jneumeth.2011.11.030

Gottschling, C., Dzyubenko, E., Geissler, M., and Faissner, A. (2016). The indirect neuron-astrocyte coculture assay: an in vitro set-up for the detailed investigation of neuron-glia interactions. J. Vis. Exp. e54757. doi: 10.3791/ 54757
Goubko, C. A., and Cao, X. (2009). Patterning multiple cell types in co-cultures: a review. Mater. Sci. Eng. C 29, 1855-1868. doi: 10.1016/j.msec.2009.02.016

Hol, E. M., and Pekny, M. (2015). Glial fibrillary acidic protein (GFAP) and the astrocyte intermediate filament system in diseases of the central nervous system. Curr. Opin. Cell Biol. 32, 121-130. doi: 10.1016/j.ceb.2015.02.004

Honegger, T., Thielen, M. I., Feizi, S., Sanjana, N. E., and Voldman, J. (2016). Microfluidic neurite guidance to study structure-function relationships in topologically-complex population-based neural networks. Sci. Rep. 6:28384. doi: $10.1038 /$ srep28384

Hughes, C. S., Postovit, L. M., and Lajoie, G. A. (2010). Matrigel: a complex protein mixture required for optimal growth of cell culture. Proteomics 10, 1886-1890. doi: 10.1002/pmic.200900758

Huh, D., Hamilton, G. A., and Ingber, D. E. (2011). From 3D cell culture to organs-on-chips. Trends Cell Biol. 21, 745-754. doi: 10.1016/j.tcb.2011.09.005

Ito, D., Tamate, H., Nagayama, M., Uchida, T., Kudoh, S. N., and Gohara, K. (2010). Minimum neuron density for synchronized bursts in a rat cortical culture on multi-electrode arrays. Neuroscience 171, 50-61. doi: 10.1016/j.neuroscience.2010.08.038

Johnson, M. A., Weick, J. P., Pearce, R. A., and Zhang, S.-C. (2007). Functional neural development from human embryonic stem cells: accelerated synaptic activity via astrocyte coculture. J. Neurosci. 27, 3069-3077. doi: 10.1523/JNEUROSCI.4562-06.2007

Jones, E. V., Cook, D., and Murai, K. K. (2012). A Neuron-astrocyte co-culture system to investigate astrocyte-secreted factors in mouse neuronal development. Methods Mol. Biol. 814, 341-352. doi: 10.1007/978-1-61779-452-0_22

Jones, E., Oliphant, T., Peterson, P., et al. (2001). SciPy: Open Source Scientific Tools for Python. Available online at: http://www.scipy.org

Jones, I. L., Livi, P., Lewandowska, M. K., Fiscella, M., Roscic, B., and Hierlemann, A. (2011). The potential of microelectrode arrays and microelectronics for biomedical research and diagnostics. Anal. Bioanal. Chem. 399, 2313-2329. doi: 10.1007/s00216-010-3968-1

Kaech, S., and Banker, G. (2006). Culturing hippocampal neurons. Nat. Protoc. 1, 2406-2415. doi: 10.1038/nprot.2006.356

Khakh, B. S., and Sofroniew, M. V (2015). Diversity of astrocyte functions and phenotypes in neural circuits. Nat. Neurosci. 18, 942-952. doi: 10.1038/nn.4043

Kidambi, S., Lee, I., and Chan, C. (2008). Primary neuron/astrocyte coculture on polyelectrolyte multilayer films: a template for studying astrocytemediated oxidative stress in neurons. Adv. Funct. Mater. 18, 294-301. doi: 10.1002/adfm.200601237

Kim, Y. H., Choi, S. H., D’Avanzo, C., Hebisch, M., Sliwinski, C., Bylykbashi, E., et al. (2015). A 3D human neural cell culture system for modeling Alzheimer's disease. Nat. Protoc. 10, 985-1006. doi: 10.1038/nprot.2015.065

Kleinman, H. K., and Martin, G. R. (2005). Matrigel: basement membrane matrix with biological activity. Semin. Cancer Biol. 15, 378-386. doi: 10.1016/j.semcancer.2005.05.004

Knight, E., and Przyborski, S. (2015). Advances in 3D cell culture technologies enabling tissue-like structures to be created in vitro. J. Anat. 227, 746-756. doi: $10.1111 /$ joa. 12257

Kuijlaars, J., Oyelami, T., Diels, A., Rohrbacher, J., Versweyveld, S., Meneghello, G., et al. (2016). Sustained synchronized neuronal network activity in a human astrocyte co-culture system. Sci. Rep. 6:36529. doi: 10.1038/srep36529

Kunze, A., Lengacher, S., Dirren, E., Aebischer, P., Magistretti, P. J., and Renaud, P. (2013). Astrocyte-neuron co-culture on microchips based on the model of SOD mutation to mimic ALS. Integr. Biol. 5, 964. doi: 10.1039/c3ib $40022 \mathrm{k}$

Levy, A. F., Zayats, M., Guerrero-Cazares, H., Qui-ones-Hinojosa, A., and Searson, P. C. (2014). Influence of basement membrane proteins and endothelial cellderived factors on the morphology of human fetal-derived astrocytes in 2D. PLoS ONE 9:e92165. doi: 10.1371/journal.pone.0092165

Lischka, F. W., Efthymiou, A., Zhou, Q., Nieves, M. D., McCormack, N. M., Wilkerson, M. D., et al. (2017). Neonatal mouse cortical but not isogenic human astrocyte feeder layers enhance the functional maturation of induced pluripotent stem cell-derived neurons in culture. Glia 66, 725-748. doi: $10.1002 /$ glia.23278

Lucius, R., and Mentlein, R. (1995). Development of a culture system for pure rat neurons: advantages of a sandwich technique. Ann. Anat. 177, 447-454. doi: 10.1016/S0940-9602(11)80152-4 
Majumdar, D., Gao, Y., Li, D., and Webb, D. J. (2011). Co-culture of neurons and glia in a novel microfluidic platform. J. Neurosci. Methods 196, 38-44. doi: 10.1016/j.jneumeth.2010.12.024

Matsusaki, M., Case, C. P., and Akashi, M. (2014). Three-dimensional cell culture technique and pathophysiology. Adv. Drug Deliv. Rev. 74, 95-103. doi: 10.1016/j.addr.2014.01.003

Missoum, K., Belgacem, M., and Bras, J. (2013). Nanofibrillated Cellulose surface modification: a review. Materials 6, 1745-1766. doi: 10.3390/ma6051745

Muramoto, K., Ichikawa, M., Kawahara, M., Kobayashi, K., and Kuroda, Y. (1993). Frequency of synchronous oscillations of neuronal activity increases during development and is correlated to the number of synapses in cultured cortical neuron networks. Neurosci. Lett. 163, 163-165. doi: 10.1016/0304-3940(93)90372-R

Odawara, A., Gotoh, M., and Suzuki, I. (2013). Control of neural network patterning using collagen gel photothermal etching. Lab. Chip 13, 2040. doi: $10.1039 / \mathrm{c} 31 \mathrm{lc} 00036 \mathrm{~b}$

O'Donovan, M. J. (1999). The origin of spontaneous activity in developing networks of the vertebrate nervous system. Curr. Opin. Neurobiol. 9, 94-104. doi: 10.1016/S0959-4388(99)80012-9

Pamies, D., Hartung, T., and Hogberg, H. T. (2014). Biological and medical applications of a brain-on-a-chip. Exp. Biol. Med. 239, 1096-1107. doi: $10.1177 / 1535370214537738$

Park, L. C. ., Zhang, H., and Gibson, G. E. (2001). Co-culture with astrocytes or microglia protects metabolically impaired neurons. Mech. Ageing Dev. 123, 21-27. doi: 10.1016/S0047-6374(01)00336-0

Pekny, M., and Pekna, M. (2014). Astrocyte reactivity and reactive Astrogliosis: costs and benefits. Physiol. Rev. 94, 1077-1098. doi: 10.1152/physrev.00041.2013

Perea, G., Navarrete, M., and Araque, A. (2009). Tripartite synapses: astrocytes process and control synaptic information. Trends Neurosci. 32, 421-431. doi: 10.1016/j.tins.2009.05.001

Perea, G., Sur, M., and Araque, A. (2014). Neuron-glia networks: integral gear of brain function. Front. Cell. Neurosci. 8:378. doi: 10.3389/fncel.2014. 00378

Placone, A. L., McGuiggan, P. M., Bergles, D. E., Guerrero-Cazares, H., Quiones-Hinojosa, A., and Searson, P. C. (2015). Human astrocytes develop physiological morphology and remain quiescent in a novel $3 \mathrm{D}$ matrix. Biomaterials 42, 134-143. doi: 10.1016/j.biomaterials.2014.11.046

Pool, M., Thiemann, J., Bar-Or, A., and Fournier, A. E. (2008). NeuriteTracer: a novel ImageJ plugin for automated quantification of neurite outgrowth. J. Neurosci. Methods 168, 134-139. doi: 10.1016/j.jneumeth.2007. 08.029

Puschmann, T. B., Zandén, C., De Pablo, Y., Kirchhoff, F., Pekna, M., Liu, J., et al. (2013). Bioactive 3D cell culture system minimizes cellular stress and maintains the in vivo -like morphological complexity of astroglial cells. Glia 61, 432-440. doi: 10.1002/glia.22446

Pyka, M., Busse, C., Seidenbecher, C., Gundelfinger, E. D., and Faissner, A. (2011). Astrocytes are crucial for survival and maturation of embryonic hippocampal neurons in a neuron-glia cell-insert coculture assay. Synapse 65, 41-53. doi: 10.1002/syn.20816

Ravi, M., Paramesh, V., Kaviya, S. R., Anuradha, E., and Solomon, F. D. P. (2015). 3D Cell culture systems: advantages and applications. J. Cell. Physiol. 230, 16-26. doi: 10.1002/jcp. 24683

Ricoult, S. G., Goldman, J. S., Stellwagen, D., Juncker, D., and Kennedy, T. E. (2012). Generation of microisland cultures using microcontact printing to pattern protein substrates. J. Neurosci. Methods 208, 10-17. doi: 10.1016/j.jneumeth.2012.04.016

Ricoult, S. G., Thompson-Steckel, G., Correia, J. P., Kennedy, T. E., and Juncker, D. (2014). Tuning cell-surface affinity to direct cell specific responses to patterned proteins. Biomaterials 35, 727-736. doi: 10.1016/j.biomaterials.2013.10.023

Roy, J., Kennedy, T. E., and Costantino, S. (2013). Engineered cell culture substrates for axon guidance studies: moving beyond proof of concept. Lab. Chip 13, 498-508. doi: 10.1039/c2lc41002h
Sandström, J., Eggermann, E., Charvet, I., Roux, A., Toni, N., Greggio, C., et al. (2017). Development and characterization of a human embryonic stem cellderived 3D neural tissue model for neurotoxicity testing. Toxicol. Vitr. 38, 124-135. doi: 10.1016/j.tiv.2016.10.001

Schindelin, J., Rueden, C. T., Hiner, M. C., and Eliceiri, K. W. (2015). The ImageJ ecosystem: an open platform for biomedical image analysis. Mol. Reprod. Dev. 82, 518-529. doi: 10.1002/mrd.22489

Schutte, R. J., Xie, Y., Ng, N. N., Figueroa, P., Pham, A. T., and O'Dowd, D. K. (2018). Astrocyte-enriched feeder layers from cryopreserved cells support differentiation of spontaneously active networks of human iPSC-derived neurons. J. Neurosci. Methods 294, 91-101. doi: 10.1016/j.jneumeth.2017.07.019

Shi, M., Majumdar, D., Gao, Y., Brewer, B. M., Goodwin, C. R., McLean, J. A., et al. (2013). Glia co-culture with neurons in microfluidic platforms promotes the formation and stabilization of synaptic contacts. Lab. Chip 13, 3008-3021. doi: $10.1039 / \mathrm{c} 31 \mathrm{lc5} 249 \mathrm{j}$

Smith, G. M., Rutishauser, U., Silver, J., and Miller, R. H. (1990). Maturation of astrocytes in vitro alters the extent and molecular basis of neurite outgrowth. Dev. Biol. 138, 377-390. doi: 10.1016/0012-1606(90)90204-V

Tang, X., Zhou, L., Wagner, A. M., Marchetto, M. C. N., Muotri, A. R., Gage, F. H., et al. (2013). Astroglial cells regulate the developmental timeline of human neurons differentiated from induced pluripotent stem cells. Stem Cell Res. 11, 743-757. doi: 10.1016/j.scr.2013.05.002

Terrasso, A. P., Pinto, C., Serra, M., Filipe, A., Almeida, S., Ferreira, A. L., et al. (2015). Novel scalable 3D cell based model for in vitro neurotoxicity testing: combining human differentiated neurospheres with gene expression and functional endpoints. J. Biotechnol. 205, 82-92. doi: 10.1016/j.jbiotec.2014.12.011

Terrasso, A. P., Silva, A. C., Filipe, A., Pedroso, P., Ferreira, A. L., Alves, P. M., et al. (2017). Human neuron-astrocyte $3 \mathrm{D}$ co-culture-based assay for evaluation of neuroprotective compounds. J. Pharmacol. Toxicol. Methods 83, 72-79. doi: 10.1016/j.vascn.2016.10.001

Tomba, C., and Villard, C. (2015). Brain cells and neuronal networks: encounters with controlled microenvironments. Microelectron. Eng. 132, 176-191. doi: 10.1016/j.mee.2014.10.007

Vandamme, E. J., De Baets, S., Vanbaelen, A., Joris, K., and De Wulf, P. (1998). Improved production of bacterial cellulose and its application potential. Polym. Degrad. Stab. 59, 93-99. doi: 10.1016/S0141-3910(97)00185-7

Wang, X. F., and Cynader, M. S. (1999). Effects of astrocytes on neuronal attachment and survival shown in a serum-free co-culture system. Brain Res. Protoc. 4, 209-216. doi: 10.1016/S1385-299X(99)00019-7

Xie, Y., Schutte, R. J., Ng, N. N., Ess, K. C., Schwartz, P. H., and O’Dowd, D. K. (2017). Reproducible and efficient generation of functionally active neurons from human hiPSCs for preclinical disease modeling. Stem Cell Res. 26, 84-94. doi: 10.1016/j.scr.2017.12.003

Yang, I. H., Co, C. C., and Ho, C.-C. (2005). Spatially controlled coculture of neurons and glial cells. J. Biomed. Mater. Res. A 75A, 976-984. doi: 10.1002/jbm.a.30509

Yoo, S. J., and Nam, Y. (2012). Neurons on parafilm: versatile elastic substrates for neuronal cell cultures. J. Neurosci. Methods 204, 28-34. doi: 10.1016/j.jneumeth.2011.10.0

Conflict of Interest Statement: The authors declare that the research was conducted in the absence of any commercial or financial relationships that could be construed as a potential conflict of interest.

Copyright $@ 2018$ Aebersold, Thompson-Steckel, Joutang, Schneider, Burchert, Forró, Weydert, Han and Vörös. This is an open-access article distributed under the terms of the Creative Commons Attribution License (CC BY). The use, distribution or reproduction in other forums is permitted, provided the original author(s) and the copyright owner are credited and that the original publication in this journal is cited, in accordance with accepted academic practice. No use, distribution or reproduction is permitted which does not comply with these terms. 